\title{
Integration of Pediatric Mental Health in General Pediatrics in Eritrea: Improving Access and Collaboration
}

Paramjit T. Joshi, MD; Solomon Haile MD; Fitsum Gebremichael, MD; Aumna Aden, BA; Margot Anderson, MD

Orotta National Referral Pediatric Hospital, Asmara, Eritrea

Abstract

Background: Recognition of mental health problems among children continues to be a diagnostic challenge especially in resource poor countries where integration of such services within the primary health care framework has not been fully successfully.

Objective: To document the prevailing mental services among children in Eritrea

Methods: prospective descriptive study on policies, practices and interventions targeted at improvement of mental health services among children in Eritrea.

Results: The Pediatric Residency Programme established at Orotta School Postgraduate School of Medicine developed a curriculum which was sensitive to recognition and management of mental disorders among children. Of a total of 42 children with mental health disorders, one third had anxiety disorders, 30\% had pervasive developmental disorders one quarter psychosomatic disorders and $10 \%$ had behaviour disorders predominantly attention deficit disorder. Two illustrative cases have been presented to highlight the challenges and the requisite solutions.

Conclusion: The establishment of the Pediatric residency with a dedicated curriculum to address mental health services has contributed to the awareness and integration of such services with primary health care in Eritrea.

\section{Introduction}

We have become increasingly aware of the ways in which psychological factors modify an individual's responses to experience, including illnesses. Whenever an illness alters a child's feeling of well-being or changes the way in which his/her parents or others feel about the child, there may be psychological disturbances. These effects can be minimized or prevented through anticipatoryguidance. Additionally, many psychological disturbances first present during childhood and are often first discussed with a pediatrician. This presents an opportunity for general pediatricians to be the first line in the early recognition of potentially serious mental health problems and the promotion of positive adjustment.

Access to mental health services for those in need is challenging in many countries due to complex and often fragmented systems of care. A Report of the Surgeon General documents the high prevalence of mental health needs of America's youth, with 1 in 5 children in the United States suffering from a diagnosable mental disorder, and only $20 \%$ to $25 \%$ of affected children receiving treatment (1). This is a troubling statistic, especially when considering that treatment of many mental disorders has been deemed highly effective. No statistics are available for Eritrea.

Without intervention, child and adolescent psychiatric disorders frequently continue into adulthood. For example, research shows that when children with coexisting depression and conduct disorders become adults, they tend to use more health care services and have higher health care costs than other adults. If the system does not appropriately screen and treat them early, these childhood disorders may persist and lead to a downward spiral of school failure, poor employment opportunities, and poverty in adulthood 2. On the other hand, early identification and treatment of children with mental health problems has the potential to reduce the burden of mental illness and its many consequences. Furthermore, data from a number of sources have demonstrated that enhanced access to outpatient mental health services is costeffective (3). Shortages of children's mental health professionals make the coordination of care between pediatricians and mental health professionals essential. By addressing the administrative, financial, and communication barriers we face, we hope to improve access, collaboration, and coordination for pediatric mental health care.

The purpose of this article is to describe some examples in which pediatric consultation at the Orotta Children's Hospital led to a successful collaboration with mental health professionals; to discuss the management of clinical cases from different perspectives; and to identify specific challenges related to pediatric-psychiatry collaboration, and to generate potential solutions to these challenges.

\section{Mental Health Services in the Pediatric Setting:}

With the appropriate training and collaborative relationships, pediatricians can and should deliver mental health services to children and adolescents in the primary care setting. This setting is ideal for initiating services to children with emerging developmental and behavioral problems and common mental health disorders such as attention-deficit/hyperactivity disorder (ADHD), depression, anxiety disorders, and substance use. The primary care setting provides opportunities for early identification and intervention, counseling, guidance, care coordination, and chronic illness management. Pediatric mental health care should be friendly to families and fully coordinated with the child's other health care. The presence of a child and adolescent psychiatrist and/or other mental health professionals in the primary care setting can further expand the range of provided services. Furthermore, enhancing access to outpatient mental health services reduces 
psychiatric hospitalizations and does not significantly increase the overall cost of mental health care $(3,4)$. Children whose problems do not improve with initial intervention and/or children with more severe degrees of impairment or complex coexisting conditions require mental health specialty consultation and, often, specialty treatment.

Even after referral to or consultation with a mental health professional, the primary care clinician plays a critical role in ongoing communication and co-management to monitor the child's progress, support the child and family, and ensure coordination of care. The pediatrician can provide family-centered, coordinated, collaborative care that addresses the child holistically, which is particularly critical for children with a complex constellation of needs including serious medical illnesses (6). In some circumstances, such as a disaster or terrorist event, or residence in communities with high rates of posttraumatic stress disorder or high-risk inner city or rural areas, the need for mental health services may be far greater than the services available. For many, if not most, children affected by a critical event or those who reside in these communities, pediatricians may be the only practitioners available to address children's mental health needs.

\section{Underlying principles of comprehensive care:}

It is important to endorse the following principles:

$\Rightarrow$ Families and children need access to mental health screening and assessment and a full array of evidence based therapeutic services to appropriately address mental health conditions. $\Rightarrow$ The identification, initial assessment, and care of mental health problems ideally take place in the child's "medical home," where he or she will benefit from the strengths and skills of the pediatrician in establishing rapport with the child and family, using the pediatrician's unique opportunities to engage children and families in mental health care without stigma.

$\Rightarrow$ Pediatricians can be trained and equipped to recognize mental health problems, to manage common mental health problems, and/or to determine when mental health specialty care or referral is indicated.

$\Rightarrow$ Pediatricians and families should ideally have access to consultation and collaboration with child and adolescent psychiatrists functioning within a coordinated mental health service system which is equipped to provide support to family members of all ages. However we recognize the shortage of mental health professionals with pediatric expertise in many parts of the world.

$\Rightarrow$ Pediatricians must be recognized as a portal of entry to the specialty mental health system and an ongoing source of care and coordination for children and adolescents in the mental health specialty system.

\section{Background and History of Mental Health Services in Eritrea:}

Prior to 2008 the only psychiatric services available in Asmara were at the St. Mary's Psychiatric Hospital located on the outskirts of Asmara, Eritrea. This facility operates a chronic inpatient service for severely mentally ill adults and an outpatient service as well. However, there are few young patients being evaluated and treated at this facility. Orotta Children's Hospital is the national pediatric referral hospital for the country of Eritrea. The hospital has 200 inpatient beds and 34 beds for the emergency ward. The hospital historically has served as a training center for nurse practitioners, nurses and associate nurses. However, since 2003 it also became the primary pediatric rotation for medical students from the Orotta School of Medicine. In January of 2008 the pediatric residency program was started with the first class of 8 doctors who were all general practitioners prior to their starting residency. Having had no medical school in Eritrea prior to 2003 all of the physicians in Eritrea had received their medical training abroad - primarily in Ethiopia, Sudan, and a few European countries. During their medical school years these physicians had little exposure to psychiatry but more importantly little or no exposure to child psychiatry.

The pediatric residency program is a 2 year training program that was established by "The Partnership for Eritrea". This is an innovative collaboration between Physicians for Peace (PFP), The George Washington University Medical Center (GWUMC) and the State of Eritrea Ministry of Health. To meet Eritrea's health care needs, the Eritrean Minister of health in 2006 invited PFP and GWUMC to develop and implement a graduate medical education program at the Orotta School of Medicine in Asmara and embarked on an ambitious program to establish graduate medical education and residency programs - first in pediatrics and surgery.

The aims of this partnership for Eritrea were to a) Train physicians in needed specialties through the graduate medical education programs b) Build human resource capacity and thereby improve the physician/ population ratio c) Ensure sustainability by training

Eritrean physicians to be medical educators and health care leaders D) Minimize brain drain by encouraging Eritrean physicians to stay in-country by providing educational and professional opportunities and lastly e) To apply the lessons leaned with the Eritrean success to other nations and to use this partnership as a model for other developing countries with scarce human resources.

A very important aspect of the training program in pediatrics is the teaching and mentoring that is provided by the visiting faculty primarily from the United States in various disciplines of pediatrics including child psychiatry. This model has been highly successful in promoting knowledge base, evidence based practices, professionalism, and the basic principles of conducting clinical research.

Within the first one year since the inception of the residency training program in pediatrics there have been notable, palpable and visible changes on overall patient care. As a result of this training program a comprehensive clinical program was established taking into consideration both the medical and psychological needs of every pediatric patient seen at the Orotta pediatric hospital. This important collaboration was 
brought about by efforts made to incorporate training and skill building into the regular medical curriculum of the pediatric residents.

\section{Psychiatry curriculum:}

Both in early 2008 a board certified child and adolescent psychiatrist came as a visiting faculty member to the Orotta School of Medicine. Given that this was the first visiting faculty member there were trepidations as to how a child psychiatrist would be received and viewed by the patients and their families in Eritrea. However, there were also concerns about whether or not child mental health would be considered an important priority by the pediatric residents who had just embarked on their 2 year pediatric residency program. The curriculum included the child psychiatrist's being present in daily morning rounds where children admitted in the past 24 hours were discussed by the entire team of residents, medical students rotating through pediatrics and the pediatric faculty.. This allowed the clinical presentation of each case discussed to also take into account any psychological factors that ought to have been considered in the patient's presentation and treatment plan. Further, the pediatric residents worked along side the child psychiatrist to interview patients who the team thought may have a psychological/emotional factor that impeded their care or were viewed to be critical in the total management of the patient.

Over a short span of 2 weeks more than 30 patients were seen for possible mental health issues - the vast majority having to do with psycho-somatic illnesses or psychological factors impacting chronic medical illnesses, such as headaches, stomach aches, attentional problems, seizures, sleep difficulties, family conflicts to just name a few. Concerns about the pediatric residents' not valuing the training in child mental health proved to be unfounded!

As part of the didactic curriculum daily lectures were given followed by a case discussion to the pediatric residents and medicals students. There were lectures given on: An introduction to the perspectives of psychiatry, Attention deficit Hyperactivity Disorder (ADHD), Behavioral disorders, Psychotic Disorders, Post Traumatic Stress Disorder (PTSD), Depression and Suicide, Psychopharmacology, Psychological factors impacting medical illness. The senior medical students in addition had daily tutorials where each of them took turns to present a case of a patient with mental health issues that they had identified and would present the history, mental status examination findings, discuss a formulation, treatment plan and prognosis. At the end of the two weeks all of the residents and students took an in-service multiple choice examinations, based on the curriculum that had been developed and taught. After the visiting faculty departed communication continued with the residents via email. Cases were discussed and consultation sought as how best to manage a particular child in question. Patients continued to be discussed and seen in the following venues: cases discussed in morning rounds; patients seen in the outpatient department, neurology clinic, and inpatient wards; and referrals from the staff psychologist at Orotta Children's Hospital.

A year later the same faculty member returned for another 2 weeks. The format of the morning rounds, case discussions, and didactic seminars continued. Topics covered in the second year included, sleep disorders, communication disorders, psychological testing, expressive therapies, attachment, bullying and adolescent development.

During the twelfe months sinc the inception of the Pediatric Residency Training programme, 42 cases of mental disorders among children were diagnosed amnd managed. The commonest disorder was anxiety disorder followed by psychosomatic disorder. (Figure 1).

Figure 1: Children with specified mental health disorders 2008-2009

\section{Number of children with specified mental disorderx. 2003-2009 (12 monthes)}

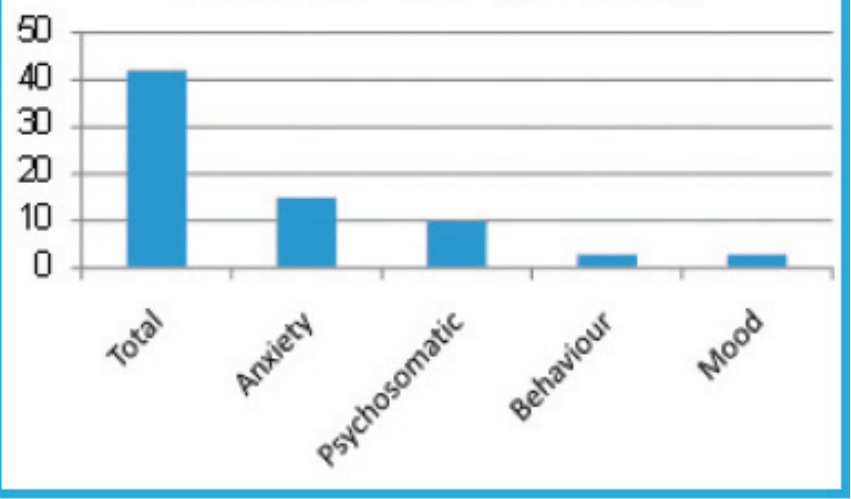

Table 6 reveals whether Traditional birth attendants recommend postpartum visit to a health facility for those deliveries they attended and when they do, the timing they recommended for the visit. Findings indicates only a cumulative of 14 percent routinely advise mothers to visit a health facility for postpartum check up with in the first 6 weeks after delivery. The remaining majority (85 percent) do not advise mothers to go for a postpartum checkup routinely. Sixteen percent advise mothers to go to a health facility for a postpartum check up only if they encountered a problem during delivery or if the mother is ill, while two third of the TBAs never advise mothers to go to health facility for a post partum check up. The proportion of TBAs who never advise or refer mothers to a health facility for a postpartum check up is higher among the untrained TBAs (77 percent) than the trained TBAs (60 percent)

\section{Therapeutic interventions used included:}

Early identification, Individual therapy, Rela xation techniques, Expressive therapy, Play therapy, Family therapy, Behavioral management, Environmental manipulation, School intervention and Pharmacotherapy

Two cases are described below that highlight the need for incorporating mental health into primary pediatrics and the benefit of doing so. 


\section{Patient 1: KA}

History: This is a 13.5 yrs old girl who has a history of Insulin Dependent Diabetes Mellitus (IDDM) of three years duration. She presented to the Emergency Department of the Orotta Children's Hospital, as a selfreferral, and related her own history to the Pediatric resident. It is to be noted that she at first wanted the physician to think that she had been brought by her father. Psycho-social history: Her mother died a year ago due to an illness that was not specified, and she has a sister who is four years younger. Recently their father re-married and brought the step-mother to live with the family - she was initially introduced as a relative. This fact is extremely important in the history of this patient since she stressed it over and over again over the course of her treatment. HPI: When the patient presented in the ED her presenting complaint was that she felt weak and that she was under insulin therapy. She gave no history that could have been construed as a precipitating factor to this event. She was kept in the ED overnight and was treated with $50 \%$ glucose and IV fluids. Once stabilized, she was transferred to the inpatient ward. Once in the ward, she was so familiar and at ease managing all routine activities but her blood sugar was on the higher side at all times. She never had a worried look on her face, in fact she appeared euphoric, replied with a broad smile at morning rounds and looked happy rather than sad being in the hospital in her interactions with the other children, mostly younger, and their families including older teens to whom she befriended with great ease.

Developmental history was unremarkable and her milestones were reported to have been achieved at the normal age periods. She was up-to-date with her immunizations and there was no past psychiatric history. She was an average student in the local school and not much was known about her peer relationships. Family history was negative for any known psychiatric illness.

Her first admission was on 2/13/06 when she first diagnosed to have diabetes. After 21 days in the hospital her blood sugar was stabilized on a combination of rapidly acting and intermediate insulin, of 26 I.U. Her weight was $34 \mathrm{~kg}$. She improved remarkably and was discharged home. Her second admission was on 12/10/06 with poor control of her diabetes. She stayed for 18 days and was discharged after she improved with weight of $37 \mathrm{~kg}$ with $54 \mathrm{IU}$ of insulin. Third admission, a year later, was on 12/25/07 with poor diabetic control, weight of $42 \mathrm{~kg}$. She had a 30-day hospital stay and was discharged improved with a total dose of $45 \mathrm{IU}$ of insulin.

She showed up in the ED again a year later on 11/7/08 with poor control of her diabetes, weighing 42 $\mathrm{kg}$ and was transferred to the ward the following day. The close follow up of her general condition was from 14 th to the 19th. Her stay was only 12 days. This was achieved by encouraging her to stick to appropriate feeding pattern and activities. It was explained to her that she will be given the accurate insulin dose and administration by the health personnel only. This was to compare her self injection accuracy and compliance.
She accepted this intervention gracefully and after she was further counseled about her diabetic care she was discharged home with an insulin dose of 44 IU.

The treatment team asked her to see her family and speak to them. She agreed but no body came and it was recalled that no visitors ever came to see her. She was observed to be by her self at all times. She explained that her sister came every evening after school to visit her and brought some food on those occasions. Her father was assumed unable to come due his working conditions even though her home was very close to the hospital. At one point she was found in the ward sharing her bed with another patient who was close to her age. She gave all kinds of reasons that did not make much sense about why she was sharing the bed with this other girl. Then the patient left the hospital unnoticed by any of the staff. Later it was learned that this other girl who was a newly diagnosed diabetic from around Asmara was crying because she was missing her things - some sugar and 'tihni' and it was concluded that our patient must have taken them from her without being noticed. This explained two things - her repeated poorly controlled diabetes leading to multiple admissions and a tendency for abusive/ unusual behaviors. The head nurse decided to talk to the patient and inquire after her. The treatment team agreed not to frustrate her by challenging her to make matters worse.

But to the surprise of the treatment team, she was admitted to the ED on the same day that she left. She was brought in by her father with the complaint of weakness. Her blood sugar at the time was 216 . She was even started on IVF and Ampicilline injections. The next day she was seen wandering around the hospital instead of resting. She explained that she was waiting for her 'family' to come and take her home. She would sit at the steps of the ED sullen and withdrawn, unusual for her, and she would immediately reply that her sister was coming soon. It seemed that she had regrets about something and that she was deeply concerned. She was assessed to be stable at the time. Again the following day she was still at the ED and her intentions were unclear. Repeat blood sugar results were between 400 and 500. It was then decided to admit her again to the ward.

Quickly the pediatric resident informed the psychologist about the situation. Jointly the resident and the psychologist decided to counsel her then and there. The interview was welcomed by the patient, still on her own with no family available to give consent. The team had to be very gentle and considerate wanting desperately to help her by finding out the core problem for these unnecessary admissions and her helpless attitude. She finally related her story openly and relieved herself of pent-up emotions. She was openly weeping, and spoke in a clear voice. She was concerned about her younger sister whom she felt was not being treated right by her step-mother. After discussing these things with the psychologist she looked visibly relieved. She thanked the psychologist and pediatric resident before leaving.

Recommendations and treatment plan continued 